\title{
ANÁLISE DO CONCEITO DE INSUCESSO DA CATETERIZAÇÃO INTRAVENOSA PERIFÉRICA EM CRIANÇAS HOSPITALIZADAS
}

\author{
$\underline{\text { John Almeida Damasceno }}{ }^{1}$; Luciano Marques dos Santos $^{2}$; Cleonara Sousa Gomes \\ e Silva $^{3}$; Erika Anny Costa Cerqueira ${ }^{4}$ \\ 1. Voluntário do Programa de Estágio Voluntário de Iniciação Científica. Estudo vinculado ao Projeto "Segurança do paciente \\ pediátrico e sua família: estudo de tecnologias e eventos adversos associados à terapia intravenosa periférica", Departamento de \\ Saúde, Universidade Estadual de Feira de Santana, e-mail: johndamasceno05@gmail.com \\ 2. Orientador e coordenador do projeto "Segurança do paciente pediátrico e sua família: estudo de tecnologias e eventos adversos \\ associados à terapia intravenosa periférica", Departamento de Saúde, Universidade Estadual de Feira de Santana, e-mail: \\ lucmarxenfo@yahoo.com.br \\ 3. Voluntária do Programa de Estágio Voluntário de Iniciação Científica. Bolsista PIBEX. Departamento de Saúde, Universidade \\ Estadual de Feira de Santana, e-mail: cleosilvauefs@gmail.com \\ 4. Voluntária do Programa de Estágio Voluntário de Iniciação Científica. Bolsista PIBEX. Departamento de Saúde, Universidade \\ Estadual de Feira de Santana, e-mail: anny c@live.com
}

PALAVRAS-CHAVE: Cateterismo periférico. Criança hospitalizada. Formação de conceito.

\section{INTRODUÇÃO}

A Cateterização Intravenosa Periférica (CIP) tem sido o procedimento mais comumente utilizado na terapêutica clínica de crianças hospitalizadas para $\mathrm{o}$ estabelecimento da Terapia Intravenosa (TIV). Entretanto, dependendo das condições da rede venosa da criança, os trabalhadores da saúde terão dificuldades para o estabelecimento de um acesso venoso periférico, gastando muito tempo executando diversas tentativas de CIP, o que provocará estresse, dor e sofrimento à criança.

Esse não estabelecimento do acesso venoso pode ser conhecido como insucesso. Alguns autores o definem como a não realização da cateterização intravenosa periférica na primeira tentativa (AVELAR et al., 2013; LARSEN et al., 2010; CARR et al., 2015) ou quando é necessário realizar mais que três tentativas (WITTING, 2011). Sendo assim, observa-se que não há definição única e padronizada para esse evento, o que resulta na necessidade de estabelecer e clarificar este conceito.

Isto posto, na tentativa de responder ao seguinte questionamento: "que conceito pode ser atribuído ao insucesso na CIP periférica em crianças hospitalizadas à luz da literatura nacional e internacional e expertise clínica de trabalhadores da saúde?”, o objetivo deste trabalho consiste, assim, em analisar o conceito de insucesso da CIP em crianças hospitalizadas.

\section{MATERIAIS E MÉTODOS}

Para a análise do conceito de insucesso na CIP, foi realizado um estudo descritivo, exploratório, metodológico e qualitativo, tendo como referencial teórico e metodológico o método híbrido. Esse método interliga análise teórica e observação empírica, sendo dividido em três fases: fase teórica, fase de campo e fase analítica. Em cada uma destas etapas, busca-se compor as quatro categorias de análise de conceito: atributos, antecedentes, consequências e definição do conceito (SCHWARTZBARCOTT; KIM, 2000).

$\mathrm{Na}$ fase teórica, foi realizado um levantamento do estado da arte, em bases de dados nacionais e internacionais, sobre o insucesso da CIP. Na segunda fase, que consiste no trabalho de campo, foram realizadas entrevistas semiestruturadas com 10 profissionais que atuam num hospital público pediátrico do interior da Bahia. Estes participaram da entrevista mediante a assinatura do Termo de Consentimento Livre e 
Esclarecido. As questões que compuseram a entrevista se relacionavam ao insucesso na CIP.

A fase analítica, por fim, consistiu na etapa de articulação dos resultados das fases teórica e de campo, e permitiu a caracterização dos componentes do conceito de forma mais abrangente (SOUZA; VERISSIMO, 2015).

Esta pesquisa respeitou os aspectos éticos da Resolução no 466/12 do Conselho Nacional de Saúde (BRASIL, 2012) e encontra-se vinculada ao projeto "Segurança do paciente pediátrico e sua família: estudo de tecnologias e eventos adversos associados à terapia intravenosa periférica", aprovado através do parecer de nº 841.612 pelo Comitê de Ética em Pesquisa (CEP) da Universidade Estadual de Feira de Santana.

\section{RESULTADOS}

$\mathrm{Na}$ fase teórica, foram analisados 15 artigos das literaturas nacional e internacional, publicados entre os anos de 2002 a 2015. Algumas das características citadas que influenciam o insucesso da (CIP) foram: condições da rede venosa (NAFIU et al., 2010; CONSTANTINO et al., 2005; RIKER et al., 2011;), o grau de nutrição, a idade (CUPER et al., 2012), a experiência do profissional (JACOBSON; WINSLOW, 2005), a história clínica de insucesso na CIP (YEN et al., 2008), uso anterior de PICC ou CVC, realização da CIP no turno diurno, pouca cooperação do paciente, ter pele escura, ser do sexo feminino, estar acordado e início da CIP no antebraço ao invés da fossa cubital.

Quanto aos atributos, que representam as características do insucesso na CIP, são citados: demora na realização da CIP, dificuldade na realização da CIP e diminuição da confiança da profissional que realiza o procedimento.

Com relação ao conceito de insucesso, alguns autores definem como a não realização da cateterização intravenosa periférica na primeira tentativa ou quando é necessário realizar mais que três tentativas.

$\mathrm{Na}$ fase de campo, como já foi mencionado, foram realizadas as entrevistas. As respostas obtidas foram inseridas em quatro categorias: definição de insucesso, antecedentes do insucesso, consequências do insucesso e estratégias da prática clínica diante do insucesso. Para cada categoria foram atribuídos uma unidade de significado, significado e unidade de significação.

Na categoria definição de insucesso, a unidade de significado mais prevalente 05 entrevistados - foi a de "não conseguir a CIP", cujo significado é "o insucesso é descrito como a tentativa de puncionar a veia, no entanto, não conseguir um acesso venoso periférico". Na categoria antecedentes do insucesso, foram encontradas 17 unidades de significado, sendo o "grau de nutrição do paciente" e a "hiperatividade da criança contribui para o insucesso" os significados com maior número de unidades de significado. Na categoria consequências do insucesso, o "estresse da criança" e a "interrupção do tratamento" foram as unidades de significado mais referidas pelos entrevistados. A categoria estratégias da prática clínica diante do insucesso teve no "auxílio de outros profissionais" a unidade de significado com maior número de respostas dos entrevistados, totalizando 05 entrevistados.

\section{DISCUSSÃO}

Os resultados mostram que há uma interpretação diferente quanto ao conceito de insucesso na literatura e no campo de prática. Já os antecedentes e as consequências do insucesso mostram pontos de encontro entre as duas fases estudadas (teórica e estudo de campo). 
$\mathrm{Na}$ literatura, a definição do insucesso tem no número de tentativas um dos fatores determinantes, sendo a não realização da CIP na primeira tentativa mencionada por diversos autores (AVELAR et al., 2013; LARSEN et al., 2010; CARR et al., 2015). Já nas entrevistas, apesar de dois entrevistados falarem do insucesso da CIP após a primeira tentativa, a maioria dos entrevistados definiu o insucesso como não conseguir a CIP. Isto é, não há um indicador do insucesso, é simplesmente não conseguir: "Quando a gente não consegue, né, pegar o acesso. [...] não consegue o acesso periférico. (E05) ". Isso dá margem a múltiplas interpretações e a não padronização do que é considerado erro no procedimento, levando às consequências.

Os antecedentes encontrados na literatura foram diversos, assim como nas entrevistas. O destaque é dado para características do paciente no momento da realização da CIP, como é o caso do grau de nutrição do paciente, que foi destacado nas duas fases. A desnutrição e a desidratação foram abordadas nas duas fases, como pode ser exemplificado por E07: "É isso, eu acho que essas crianças menores... Umas crianças, é... Desidratada, desnutrida, que tenham realmente uma dificuldade pra puncionar o acesso. Pra se ver a via, né, localizar a rede venosa”; os artigos ainda trazem a obesidade como outro determinante do insucesso. Essas informações são muito importantes, já que a apresentação do paciente se torna um indicador da necessidade de um profissional mais experiente para a realização da CIP.

Fica evidente que uma consequência importante, e encontrada nas duas fases do estudo, é o estresse para os pacientes, como fala E09: "Eeee. Eu acho que tanto psicológica para o paciente que ele vai ser puncionado novamente que gera um estresse a mais para ele e para a família dele [...]". A fala ainda traz a repercussão desse estresse se estendendo para a família do paciente e, sem dúvidas, o estresse também é repercutido nos profissionais que realizam a CIP, leva o paciente a sentir mais dor (MALYON et al., 2014) e gera complicações como infecções, hematoma, flebite, contusões da pele, danos nos nervos e transfixação dos vasos. Outro ponto que chamou atenção como consequência do insucesso foi que um entrevistado não reconheceu consequências para esse evento: "Quais conseqüências atribui ao insucesso? E tem conseqüências? Eu acho que não. Não tem conseqüências ao insucesso. Você me pegou agora viu!?" (E03).

\section{CONCLUSÃO}

Como representações de uma dada realidade, os conceitos possuem atributos de caráter dinâmico, mutáveis na dimensão temporal e contextual, sendo sua evolução influenciada pelo uso e aplicação. Dessa forma, para cumprir seu papel, a análise de conceito do insucesso na CIP deve aprimorar um conceito já introduzido e definido na literatura, de modo a torna-lo aplicável eficazmente na pesquisa e na prática clínica (WALKER; AVANT, 1995).

\section{REFERÊNCIAS}

AVELAR, A. F. M. et al. Assertividade e tempo de permanência de cateteres intravenosos periféricos com inserção guiada por ultrassonografia em crianças e adolescentes. Rev Esc Enferm USP., v. 47, n. 3, p. 539-546. 2013.

BRASIL, Ministério da Saúde. Conselho Nacional de Saúde. Resolução 466 de 12 de dezembro de 2012: diretrizes e normas regulamentadoras da pesquisa envolvendo seres humanos. Brasília, 2012. Disponível em: 
<http://bvsms.saude.gov.br/bvs/saudelegis/cns/2013/res0466_12_12_2012.html>. Acesso em: 15 mai 2017.

CARR, PETER J. et al. Insertion of peripheral intravenous cannulae in the Emergency Department: factors associated with first-time insertion success. Journal of Vascular Access, v. 17, n. 2, p. 182-190. 2015.

CONSTANTINO, THOMAS G. et al. Ultrasonography-Guided Peripheral Intravenous Access Versus Traditional Approaches in Patients With Difficult Intravenous Access. Annals of emergency medicine, v. 46, n. 5, p. 456-461. 2005.

CUPER, NATASHA J. et al. Predictive factors for difficult intravenous cannulation in pediatric patients at a tertiary pediatric hospital. Pediatric Anesthesia, v. 22, p. 223229. 2012.

JACOBSON, ANN F.; WINSLOW, ELIZABETH H. Variables influencing intravenous catheter insertion difficulty and failure: an analysis of 339 intravenous catheter insertions. Heart \& Lung, v. 34, n. 5, p. 345-359. 2005.

LARSEN, PAMELA. et al. Pediatric peripheral intravenous access: does nursing experience and competence really make a diference? Journal of Infusion Nursing, v. 33, n. 4, p. 226-235. 2010.

NAFIU, O. O. et al. Comparing peripheral venous access between obese and normal weight children. Pediatric Anesthesia, v. 20, p. 172-176. 2010.

RIKER, MICHAEL W. et al. Validation and refinement of the difficult intravenous access score: a clinical prediction rule for identifying children with difficult intravenous access. Academic Emergency Medicine, v. 18, p. 1129-1134. 2011.

SCHWARTZ-BARCOTT, D.; KIM, H. S. An expansion and elaboration of the hybrid model of concept development. In: RODGERS, B. L.; KNALF, K. A. Concept development in nursing: foundations, techiniques, and applications. 2nd ed. Philadelphia: Saunders; 2000.

SIMHI, ELIAHU. et al. A vein entry indicator device for facilitating peripheral intravenous cannulation in children: a prospective, randomized, controlled trial. International Anesthesia Research Society, v. 107, n. 5, p. 1531-1535. 2008.

SOUZA, J. M.; VERISSIMO, M. L. Ó R. Desenvolvimento infantil: análise deu m novo conceito. Rev. Latino-Am. Enfermagem, Ribeirão Preto, v. 23, n. 6, p. 1097-1104. 2015.

WALKER, L.O.; AVANT K.C. Concept development. In: Walker LO, Avant KC. Strategies for theory construction in nursing. 3rd ed. Norwalk: Appleton \& Lange; 1995.

WITTING, MICHAEL D. IV access difficulty: incidence and delays in an urban emergency department. The Journal of Emergency Medicine, v.42, n. 4, p. 483-487. 2012. 
YEN, KENNETH. et al. Derivation of the DIVA score :A Clinical Prediction Rule for the Identification of Children With Difficult Intravenous Access. Pediatric Emergency Care, v. 24, n. 3, p. 143-147. 2008. 